\title{
Estimation of Resident's Location in Indoor Environment Using Bioelectric Potential of Living Plants
}

\author{
Hidetaka Nambo* and Haruhiko Kimura \\ Graduate School of Natural Science and Technology, Kanazawa University, \\ Kakuma, Kanazawa, Ishikawa 920-1192, Japan
}

(Received October 30, 2015; accepted January 12, 2016)

Keywords: location estimation, plant bioelectric potential, machine learning

Because of Japan's rapidly aging population, the number of single-person households is increasing. Therefore, to ensure the safety of residents, the demand for methods of monitoring residents' behaviors and indoor situations has become higher. Instead of using devices that can violate a person's privacy, such as cameras or microphones, we focus on plant bioelectric potential for monitoring. The plant is not harmful to the resident; moreover, it may have healing effects on humans. Furthermore, previous studies show that the potential is affected by human behavior around it. Our study applies this property, and we propose a method of estimating the location of a resident in an indoor environment. In this work, we build a classification and regression model to determine the distance between plants and humans. Then, we place two or more plants in one room and estimate the distance from the resident to each plant using the built models. Then, on the basis of these estimation results, the location of the resident is estimated. Furthermore, we conduct an evaluation to compare the estimated result and the resident's location history and show the accuracy of our method.

\section{Introduction}

In Japan, it is known that the population is aging rapidly. For this reason, the number of singleperson households is increasing. Furthermore, not only for aged persons, there are also many people who live alone, away from their family. In such cases, if some accident occurs, it takes more time to detect the accident, and there is a possibility that this will affect the life of the resident because of the delay in detection. Therefore, the demand to monitor residents' behaviors to ensure their safety and security has become higher. To date, cameras or microphones are generally used for monitoring indoor environments. However, these instruments violate a person's privacy, and this is a mental burden on residents.

In this study, we focus on plant bioelectric potential as a tool for monitoring residents' behaviors. Plant bioelectric potential is a weak electric signal generated in a living plant, and it is caused by life activities of a living plant, such as a photosynthesis or transpiration, and environmental factors such as temperature or humidity around a living plant.(1) In a previous study, it was found that plant bioelectric potential is affected by human behavior or activities. Hirobayashi et al. found that when a human steps near a plant, there is a correlation between the change of plant bioelectric

"Corresponding author: e-mail: nambo@ec.t.kanazawa-u.ac.jp 
potential and the step. ${ }^{(2)}$ It is suggested that an electromagnetic wave that is generated by things like a change of a myoelectric potential caused by walking affects an electric signal in a living plant. ${ }^{(1,2)}$ Furthermore, Nomura et al. showed that human behavior, such as stepping, walking, and opening doors, can be distinguished on the basis of the difference in the plant bioelectric potential. ${ }^{(3)}$ However, these studies do not show the relationship between the change in potential and the distance of the plant from the human. In this regard, Jin et al. proposed a method of estimating the distance of a plant from a human by a machine learning method using the change in the plant's electric potential. ${ }^{(4)}$ They showed that the distance can be roughly estimated by classifying the bioelectric potential using an artificial neural network. However, only distance estimation is reported, and the report does not mention location estimation.

On the basis of these reports, we considered that it is possible to monitor a resident's location and behavior by measuring bioelectric potential. Therefore, in this work, we build a model that can estimate the distance of a plant from a human in an indoor environment by analyzing bioelectric potential using machine learning methods such as decision trees or multilayer perceptron methods. Additionally, we propose a method of estimating the location of a resident using these models in an indoor environment.

\section{Experimental Environment}

\subsection{Measurement of plant electric potential}

In this section, the measurement method of plant bioelectric potential is described. In this study, we deal with a plant's bioelectric potential. Although the potential can be observed for any living plant, we use a pothos plant for our experiment. The reasons why we chose pothos are the following. First, pothos is not expensive and easy to obtain. Second, pothos is easy to grow, so it is easy for an aged person or single, busy person to take care of it.

To observe its potential, a data logger is applied. We used the data logger GRAPHTEC GL4004 shown in Fig. 1, which can measure a very low voltage at a high sampling rate $(\sim 1 \mathrm{kHz})$. It has 4

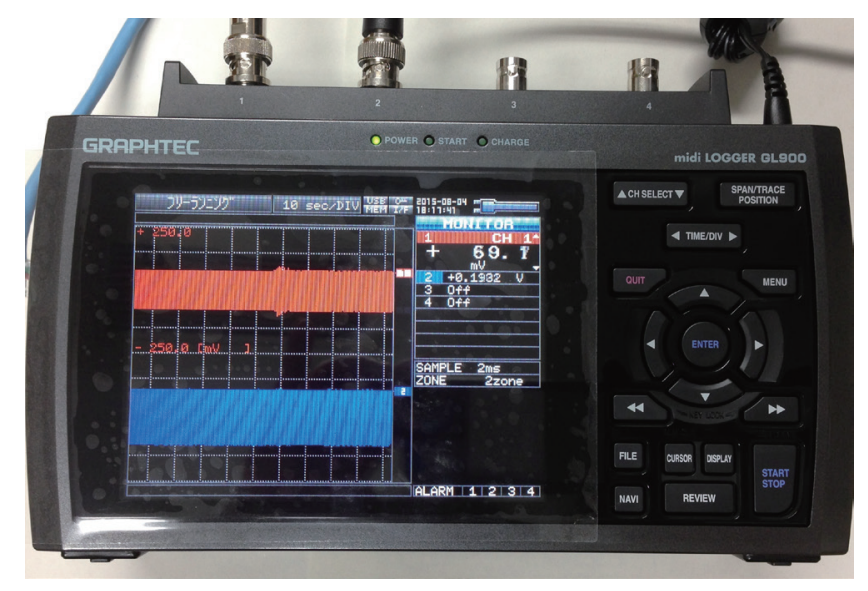

Fig. 1. (Color online) Measuring instrument; GRAPHTEC GL900-4. 
channels and it can measure 4 points of voltage simultaneously. For measuring the plant bioelectric potential, we sealed an electrode between two different leaves, and measured the voltage between the two leaves. Measured values are sent to a PC sequentially in real time via a local area network.

\subsection{Experimental Environment}

In this section, the indoor environment where the experiment was conducted is described. Figure 2 shows the layout of the indoor experimental environment. The room measures $345 \mathrm{~cm}$ by $575 \mathrm{~cm}$, and its height is $260 \mathrm{~cm}$. In the room, a desk, a table, and shelves are found. Figure 3 shows a photograph of the room. At the points $\mathrm{P} 1$ and $\mathrm{P} 2$, two pothos plants are located; one is on the table in the middle of the room, and the other is on the shelf next to the door. The points of $\mathrm{Mi}(i$ $=1-5)$ in the figure are the observation points. The coordinates of these points are shown in Table 1; the upper left position is regarded as the origin. When obtaining the data for the building estimation model, measurements were taken at these points. The details of the building model are described in $\S 3$.

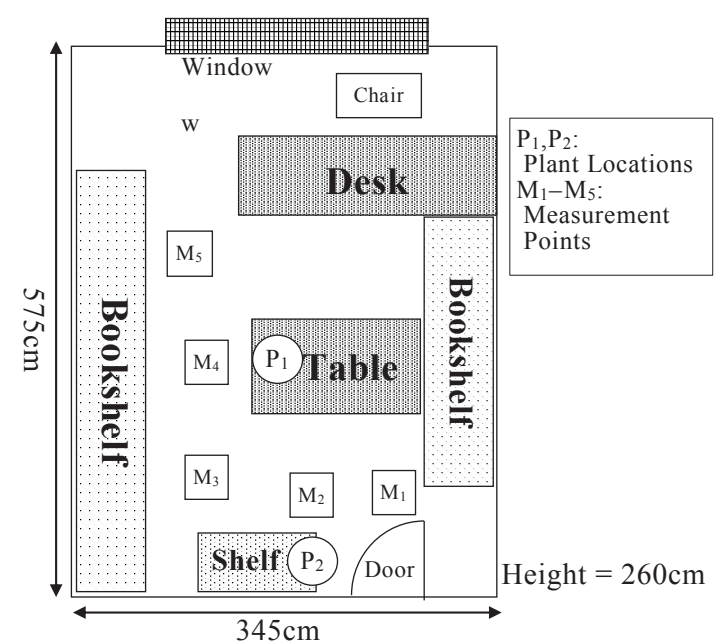

Fig. 2. Layout of the experimental environment.

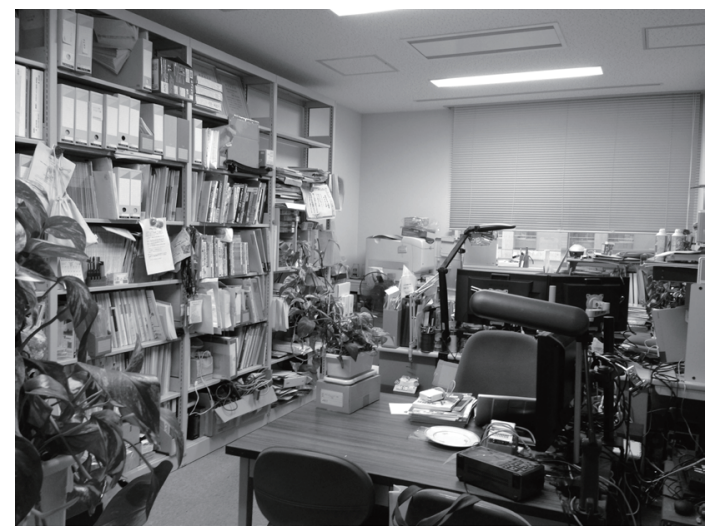

Fig. 3. A photograph of the experimental environment taken from the door.

Table 1

Location of observation points and plants.

\begin{tabular}{lcc}
\hline Observation point & $X$-coordinate & $Y$-coordinate \\
\hline M1 & 260 & 475 \\
M2 & 200 & 490 \\
M3 & 140 & 430 \\
M4 & 120 & 340 \\
M5 & 110 & 260 \\
P1 & 160 & 340 \\
P2 & 200 & 530 \\
\hline
\end{tabular}




\section{Estimation Method}

In this section, the method of estimating the location of the resident is described. The flow of the proposed method is shown in Fig. 4. Details are described as follows.

\subsection{Calculating parameters from bioelectric potentials}

All bioelectric potentials are measured at a sampling rate of $500 \mathrm{~Hz}$ and transmitted to a PC by GL900-4. Each data point is the time sequence data of a voltage. Sets of 512 sample data are regarded as 1 unit of analysis data. As shown in Fig. 5, for every 32-sample point, the last 1 unit (512 samples) of data is processed. In short, 1 unit of data is regarded as a block for analysis.

Each unit is decomposed to a smaller size for precise analysis. Figure 6 shows an original block and smaller blocks. In our method, both the smaller blocks and the original block are targets of analysis. We use various lengths of blocks to obtain various changes in the bioelectric potential.

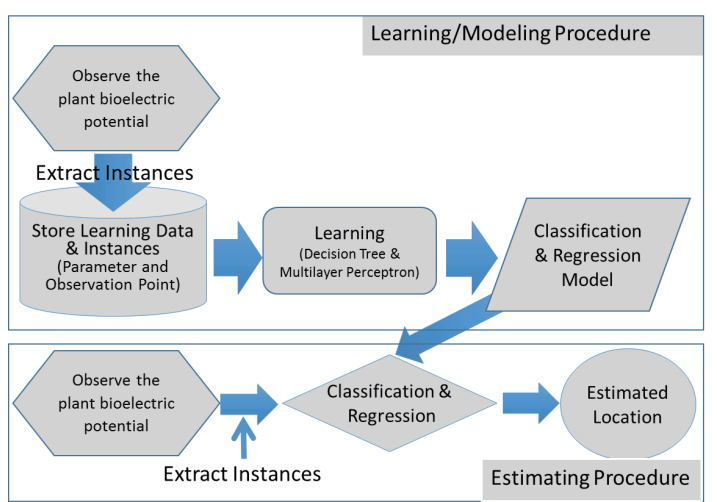

Fig. 4. (Color online) Process flow of the proposed method.

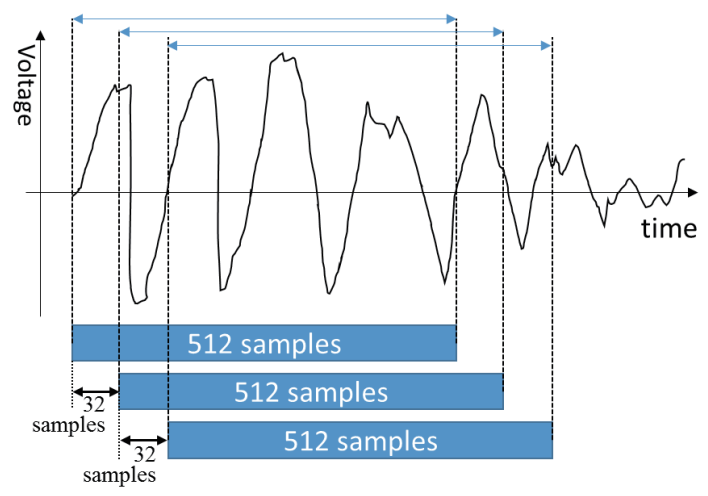

Fig. 5. (Color online) Sampling period for the bioelectric potential.

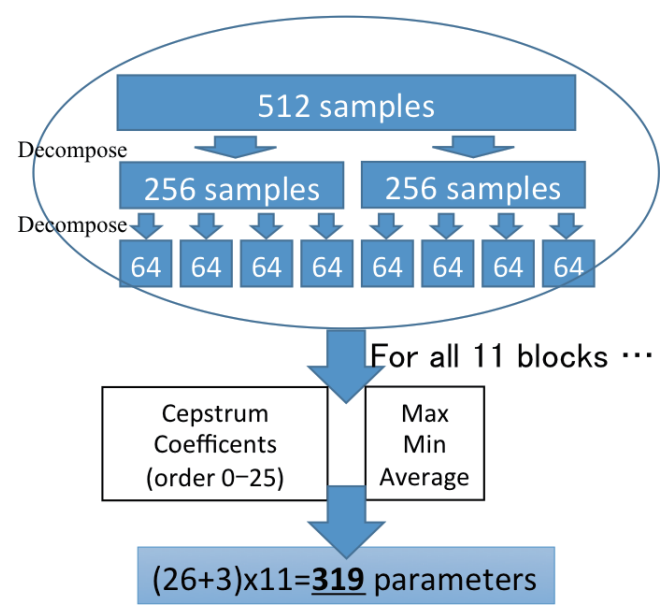

Fig. 6. (Color online) Data decomposition from a unit of analysis. 
When a resident stays near the plant for long time, the potential of the plant is affected for a long time. On the other hand, when a resident stays for a short time, such as passing by, the potential is affected for only a short time. Therefore, we have to observe various periods of the potential. In our measurement configuration, the sampling rate is $500 \mathrm{~Hz}$. In short, 500 sampling points represent the change in $1 \mathrm{~s}$. We use 512 points as a block because we consider that the potential change occurs within $1 \mathrm{~s}$ at most. For all original and decomposed blocks, cepstrum coefficients, maximum value, minimum value, and average value are calculated. Cepstrum is a result of inverse Fast Fourier Transform (iFFT) of a power spectrum of a target signal. Here, let $G(\omega)$ be a spectrum of bioelectric potential signal in steady state, let $H(\omega)$ be a spectrum of affects on bioelectric potential by human behavior, and let $S(\omega)$ be a spectrum of the observed potential affected by human behaviors. With these definitions, we have the following equation:

$$
|S(\omega)|=|G(\omega)||H(\omega)| .
$$

Taking the log of both sides of Eq. (1), we have the following equation:

$$
\log |S(\omega)|=\log |G(\omega)|+\log |H(\omega)| .
$$

A previous paper reports that the effect of human behavior on plant bioelectric potential appears at a lower band in the target signal. ${ }^{(5)}$ In short, in the frequency domain, $H(\omega)$ is distributed at a lower frequency band than $G(\omega)$. Therefor, it is considered that we can obtain the features of human behavior by decomposing $S(\omega)$ into $H(\omega)$ and $G(\omega)$. To decompose the feature from the target signal, the following procedures are carried out.

1. Let $S(t)$ be the signal of the potential.

2. Obtain a power spectrum $S(\omega)$ by performing FFT and square calculation.

3. Calculate $\log |S(\omega)|$.

4. Obtain cepstrum coefficients by calculating the inverse FFT of $\log |S(\omega)|$.

In this study, we calculated 26 cepstrum coefficients (from 0 to 25).

From each block, 29 parameters were obtained, namely, 26 cepstrum coefficients, maximum value, minimum value, and average. In total, 319 parameters were obtained from a unit of analysis, because 11 decomposed blocks were included in an original block. We regard these parameters as one instance of the unit.

\subsection{Building estimation models}

In this study, we use a decision tree and a perceptron as a learning method for an estimation model. The decision tree is for the classification model, and the perceptron is for the regression model. We collect various potential signals when the resident is at the observation point $\mathrm{M} x$, and analyze them by the method described in $\S 3.1$. Then, we obtain instances for each unit of analysis; each instance consists of 319 extracted parameters as dependent variables and the measured point $\mathrm{M} x$ (for the classification model) or the distance of the measured point $\mathrm{D} x$ (for the regression model) as target variables. They are the input data of a learning method.

We use the J48 algorithm on Weka version 3.7.12 for the decision tree method. This algorithm is used for building a decision tree, and it is based on C4.5 algorithm developed by J. R. Quinlan.(6) As a result, we obtain a classification model to estimate the measured point by a unit of potential signals. 
Furthermore, we use a multilayer perceptron on Weka version 3.7.12 for the perceptron method. As a result, we obtain a regression model to estimate the distance from plant P1 or P2 in Fig. 2.

\subsection{Classification and regression using the models}

When estimating the location, the parameters extracted from an observed unit are input to the built models. Then, the models output their estimation.

From the classification model, the classification result is the output. In this study, the model distinguishes where the input unit was observed. Options are the observation points M1-M5 and "not present in the room". Therefore, the model provides an index of 6 classes 1, 2, 3, 4, 5, and 6; they correspond to M1-M5 and "not present". On the other hand, the estimated distance is the output from the regression model. This distance is a continuous value, and it means the distance from the plant to the location of the resident.

\subsection{Estimation of location of the resident}

The procedures described in $\S \S 3.1$ and 3.2 are carried out for each channel. The number of plants in the experimental room is 2 ; thus, we obtain 2 potential signals and 2 sets of instances. Therefore, the number of sets of estimation models is also 2, and the number of model outputs, which are the set of class index and distance, is 2 . Consequently, we should derive the estimation result from 2 sets of outputs.

When estimation is conducted, the bioelectric potentials are measured and the instances are sequentially extracted from them. Then, extracted instances are put into the models one after another. In this work, the estimation result $\mathrm{R}$ is derived by the following procedure.

1. From the input of one instance, obtain class indexes using classification models and translate corresponding observation point or "not present". Let $\mathrm{C} 1$ and $\mathrm{C} 2$ be translated points.

2. If either $\mathrm{C} 1$ or $\mathrm{C} 2$ is "not present", let the estimation result $\mathrm{R}$ be "not present" and finish the procedure.

3. If $\mathrm{C} 1$ and $\mathrm{C} 2$ are the same points, then let the estimated point $\mathrm{C}$ be $\mathrm{C} 1$.

4. If both $\mathrm{C} 1$ and $\mathrm{C} 2$ are not "not present", and $\mathrm{C} 1$ is the neighbor of $\mathrm{C} 2$, then let $\mathrm{C}$ be the middle point of $\mathrm{C} 1$ and $\mathrm{C} 2$. Otherwise, let the estimated point $\mathrm{C}$ be $\mathrm{NE}$ (not estimated).

5. Obtain distances from each plant D1 and D2 using regression models.

6. Consider two circles whose center is the plant Px and whose radius is Dx. Then, calculate I1 and I2; these are their intersections.

7. Select the nearest intersection $\mathrm{I}$ from $\mathrm{C}$; then, let the estimation result $\mathrm{R}$ be the middle point of $\mathrm{C}$ and I.

8. If $\mathrm{C}$ is $\mathrm{NE}$, select the nearest intersection I from the last result $\mathrm{R}^{\prime}$, and let $\mathrm{R}$ be the middle point of $\mathrm{C}$ and $\mathrm{R}$ '. If $\mathrm{R}$ ' was not obtained, let $\mathrm{R}$ be the middle point of $\mathrm{I} 1$ and $\mathrm{I} 2$.

9. If $\mathrm{C}$ is "not present", then let $\mathrm{R}$ be "not present".

10. If no intersection is obtained, let $\mathrm{R}$ be $\mathrm{C}$.

Here, the last result $\mathrm{R}^{\prime}$ is the result for one instance before the current. 


\section{Experiments and Results}

In this section, the experiments and their results are described.

\subsection{Obtaining learning data and building estimation models}

To make the estimation models, we measure the bioelectric potentials of the plants in the room. A testee stands at the observation point and moves around the point for $30 \mathrm{~s}$. While the testee is moving, the potentials of the plants P1 and P2 are measured. After changing the observation point from M1 to M5, measurements are conducted 7 times for each point. Additionally, when the testee is out of the room, it means that the observation point is "not present", and the measurement is conducted anyway. The testee is one typical male person. In total, $210 \mathrm{~s}$ of the bioelectric potential data are obtained for each point and a plant. These data are sent to a PC sequentially and converted to instances. Then, 3171 instances are obtained for one point and one plant. In total, 19026 instances are obtained for each plant.

By applying these instances, the estimation models are built. For each plant, the J48 algorithm and the multilayer perceptron method are used to build a classification model and a regression model.

\subsection{Estimation examination}

To examine the accuracy of our proposed method, we conducted a location estimation examination. In the examination, a location trajectory was estimated from the bioelectric potential of the plant in the room using our method.

At first, a testee walked in the room for $30 \mathrm{~s}$ along a path which was defined beforehand. The testee was a male in his 40s; his height and weight were about $160 \mathrm{~cm}$ and $60 \mathrm{~kg}$. He was normally dressed and exposed little skin. When the testee was walking, the bioelectric potentials of the plant were measured and stored in the PC. After that, by analyzing these potentials by our method, the location trajectories were obtained. Then, by comparing the trajectory with the path of the testee, we evaluated our method.

We use two types of path for this experiment.

(a) The testee stood at M4 first. When the measurement started, the testee moved to M1 through M3 and M2, and then returned to M4. The testee repeated this procedure 3 times.

(b) At first, the testee waited near the chair. Ten seconds after the measurement started, the testee moved to M1 through M5, M4, M3, and M2 for $10 \mathrm{~s}$. After that, the testee went out of the room through door and waited for $10 \mathrm{~s}$.

The estimation results are shown in Figs 7-10. Figures 7 and 8 are the estimated trajectories of type (a). Figure 7 shows the $X$-coordinate of the trajectory if the upper left of the room is the origin, and Fig. 8 shows the $Y$-coordinate. To evaluate the models, Figs. 9 and 10 show the results using only the classification model. Furthermore, Figs. 11-14 are the estimated trajectories of type (b). Each figure corresponds to Figs. 7-10 of the results of type (a). 


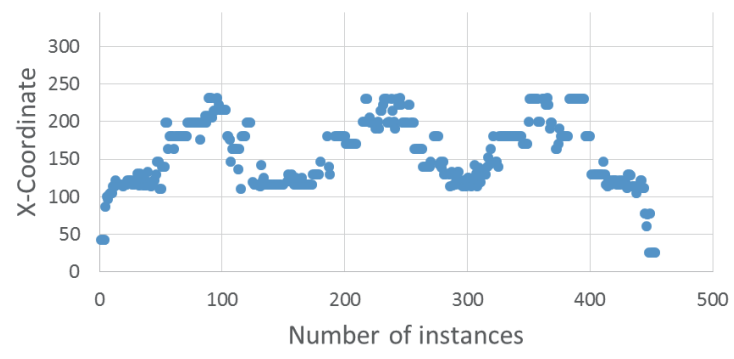

Fig. 7. (Color online) Estimation of $X$-coordinates for walking pattern (a).

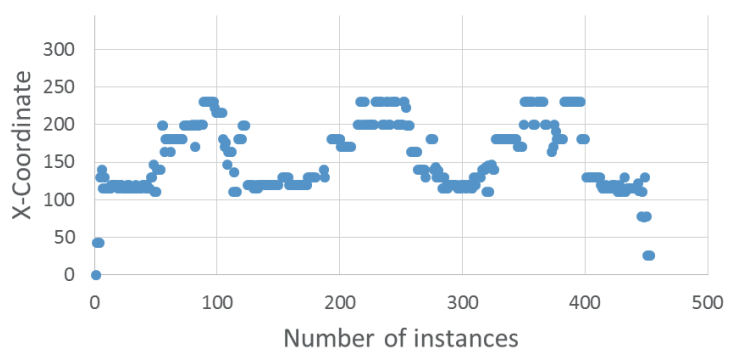

Fig. 9. (Color online) Estimation of $X$-coordinates for walking pattern (a) using only classification model.

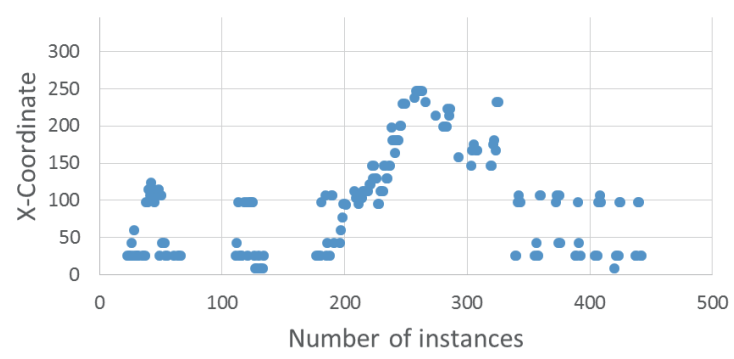

Fig. 11. (Color online) Estimation of $X$-coordinates for walking pattern (b).

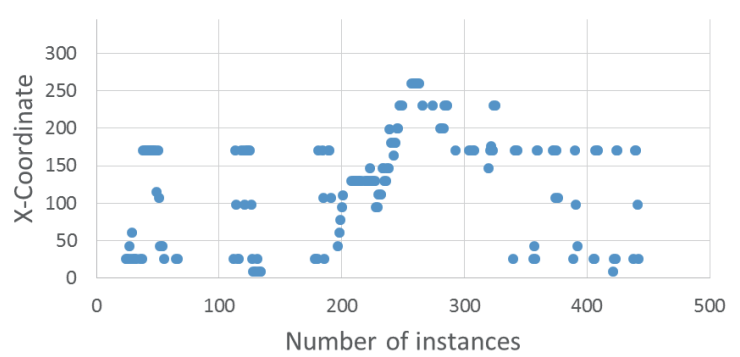

Fig. 13. (Color online) Estimation of $X$-coordinates for walking pattern (b) using only classification model.

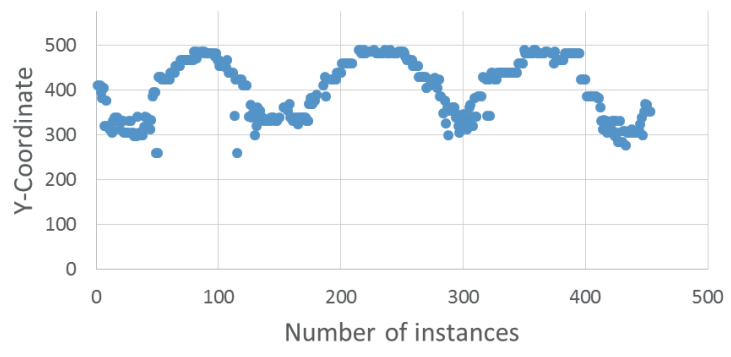

Fig. 8. (Color online) Estimation of $Y$-coordinates for walking pattern (a).

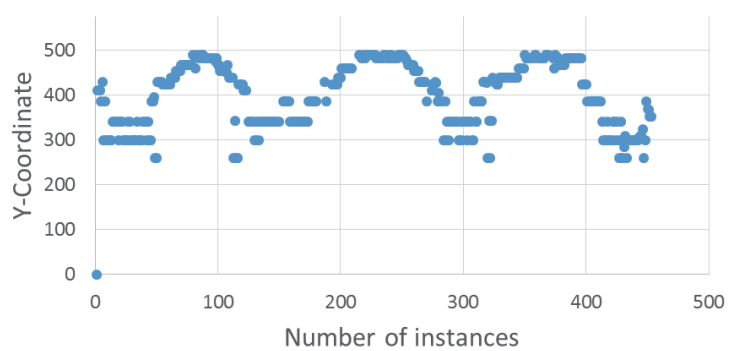

Fig. 10. (Color online) Estimation of $Y$-coordinates for walking pattern (a) using only classification model.

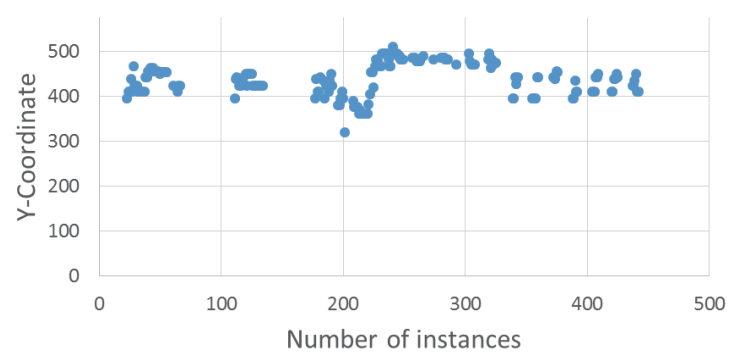

Fig. 12. (Color online) Estimation of $Y$-coordinates for walking pattern (b).

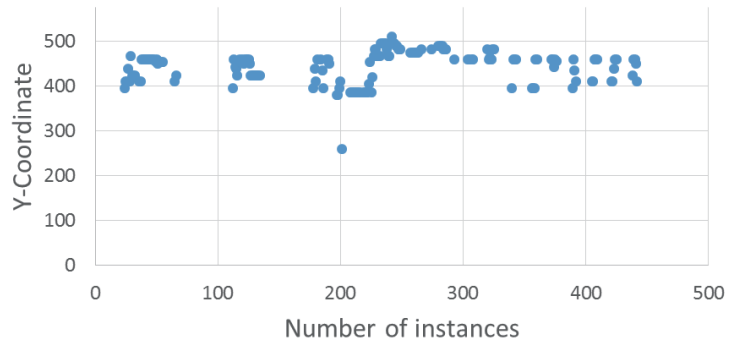

Fig. 14. (Color online) Estimation of $Y$-coordinates for walking pattern (b) using only classification model. 


\section{Discussion}

According to Figs. 7 and 8, the estimation traces the resident's movement very well. $X$ - and $Y$-coordinate changes are synchronized with the resident's movements. Furthermore, we can see that using the regression model makes the results smoother than those shown in Figs. 7 and 9 or Figs. 8 and 10. Table 2 shows the number of estimation times and the number of times that an estimation result is "not present" or "not estimated" for type (a). While conducting the experiment type (a), the testee was always in the room. Therefore, the result of "not present" or "not estimated" is an obvious error. According to Table 2, the number of errors is small. However, according to Figs. 11 and 12, the result does not trace the movement, particularly for the first $10 \mathrm{~s}$ and the last $10 \mathrm{~s}$. Table 3 shows the number of estimation times and the number of times that an estimation result is "not presnet" for the first and the last $10 \mathrm{~s}$ of type (b). During these two periods, the testee was out of the room or staying out of range of the plants. The estimation should be "not present". However, the accuracy of the result is only around $60 \%$. On the other hand, it did not estimate well the middle $10 \mathrm{~s}$, which corresponds to the number of instances of about 150 to 300 .

From this result, we consider the properties of the location estimation on the basis of the bioelectric potential. We found that the plant has a distance limit for the estimation. As shown in a previous study, ${ }^{(4)}$ when the resident is far from the plant, the effect of human movement on the plant becomes very small. In that case, the estimation does not work well. In our experiment type (b), the resident was near the chair and far from the plants for the first $10 \mathrm{~s}$, and the resident was out of the room for the last $10 \mathrm{~s}$. Even if the resident is near either plant, the estimation by the other plant does not work well. Therefore, the estimation result is poor. On the other hand, if the resident is near the plants, the estimation works well. Additionally, we found that it is difficult to estimate "not present" using the regression model. We expected that when the class cannot be estimated if classification results are different from each other, the regression model would complement the estimation. According to Figs. 13 and 14, the complementation partially works. However, when the resident was not in the room, the regression model did not provide any useful information, and the model could not determine if the resident was in the room, because the estimation model was built based on the instance when the resident was in the room.

According to this consideration, the proposed method should be improved as follows.

1. Decide the distance limit and establish a cooperative mechanism for the plants.

We should limit the range covered by one plant, and we should develop a method of informing the next plant when the resident moves beyond the range of the plant. The next plant would know where the resident comes from and it would help in the estimation.

Table 2

The number of estimation times, type of results.

\begin{tabular}{ccc}
\hline Estimation time & "Not estimated" & "Not present" \\
\hline 453 & 17 & 9 \\
\hline
\end{tabular}

Table 3

The number of estimation results for "not present".

\begin{tabular}{ccccc}
\hline \multicolumn{2}{c}{ First $10 \mathrm{~s}$} & & \multicolumn{2}{c}{ Last $10 \mathrm{~s}$} \\
\cline { 1 - 1 } Estimation times & "Not present" & & Estimation times & "Not present" \\
\hline 151 & 94 & & 151 & 87 \\
\hline
\end{tabular}




\section{Using more plants}

A plant has a distance limit; therefore, we should provide more plants in the room.

3. Using meta-knowledge

However, placing more plants is bothersome for the resident. Thus, we should consider a method of reducing the number of plants. For this problem, we should use meta-knowledge. In this study, we consider that meta-knowledge is the layout information of the room. For example, if there is a table; the resident usually will not be on it. Then, we can reduce the number of candidates of estimated locations of the resident. Moreover, if there is a chair, the possibility of the resident staying there becomes higher. Considering this information, we can build a potential map of the room. Using this map, we can reduce the number of plants while maintaining the accuracy of estimation.

\section{Conclusions}

In this paper, we proposed a method of estimating the location of a resident in an indoor environment using plant bioelectric potential. In our method, by collecting observed instances as learning data for an estimation model, we built the classification models and the regression models by applying machine learning techniques. Then, utilizing these models, the location of a resident was estimated. Furthermore, we evaluated our method by estimating location trajectories. By the evaluation, we realized that, when the resident is near a plant, our method works well; in other words, our method can estimate the location of a resident in this situation. However, our method cannot estimate well when the distance between the resident and the plant is large. In particular, it is difficult to estimate when the resident is not in the room. In future work, we will improve our method so that it can estimate more widely and accurately in an indoor environment.

\section{Acknowledgements}

This work was supported by JSPS KAKENHI Grant Number 26350066.

\section{References}

1 T. Oyabu and T. Katsube: Plant Bioelectric Potential and Communication (Kaibundou, Tokyo, 2009) pp. 1-32 (in Japanese)

2 S. Hirobayashi, Y. Tamura, Y. Yamabuchi, and T. Oyabu: Trans. Inst. Electr. Eng. Jpn. Sect. E (Japan) 127 (2007) 258 (in Japanese).

3 K. Nomura, H. Nambo, and H. Kimura: Trans. Inst. Electr. Eng. Jpn. Sect. E (Japan) 134 (2014) 206 (in Japanese).

4 X. Jin, H. Nambo, and H. Kimura: Proceedings of the 15th APIEMS (Korea 2014) pp. 602-606.

5 T. Shimbo, M. Fujii, A. Sawada, T. Oyabu, and H. Kimura: Trans. Inst. Electr. Eng. Jpn. Sect. E (Japan) 127 (2007) 425 (in Japanese).

6 J. R. Quinlan: C4.5: Programs for Machine Learning (Morgan Kaufmann Publishers, California, 1993). 